\title{
Managing hypertension in 2018: which guideline to follow?
}

\author{
Fabio Angeli, ${ }^{1}$ Gianpaolo Reboldi, ${ }^{2}$ Monica Trapasso, ${ }^{2}$ Adolfo Aita, ${ }^{3}$ \\ Paolo Verdecchia ${ }^{3,4}$
}

'Division of Cardiology and Cardiovascular Pathophysiology, Hospital 'S M della Misericordia', Perugia, Italy ${ }^{2}$ Department of Medicine, University of Perugia, Perugia, Italy

${ }^{3}$ Fondazione Umbra Cuore e Ipertensione-ONLUS, Perugia, Italy

${ }^{4}$ Struttura Complessa di Cardiologia, Hospital 'S. Maria della Misericordia', Perugia, Italy

\section{Correspondence to}

Dr Paolo Verdecchia, Fondazione Umbra Cuore e IpertensioneONLUS and Struttura Complessa di Cardiologia, Hospital S. Maria della Misericordia, Perugia, Italy; verdec@tin.it

Received 21 November 2018 Revised 30 January 2019 Accepted 3 February 2019

\section{Check for updates}

(C) Author(s) (or their employer(s)) 2019. No commercial re-use. See rights and permissions. Published by BMJ.

To cite: Angeli F, Reboldi G, Trapasso M, et al. Heart Asia 2019;11:e011127. doi:10.1136/

heartasia-2018-011127

\section{ABSTRACT}

Hypertension is a global public health issue and a major cause of morbidity and mortality. Its prevalence is increasing in many Asian countries, with a number of countries with blood pressure above the global average. Although the average systolic blood pressure is decreasing worldwide since the 1980s at the rate of about $1 \mathrm{~mm} \mathrm{Hg}$ systolic blood pressure per decade, it is increasing in low-income and middle-income countries, especially in the East and South Asian population. Of note, the much larger base Asian population results in a considerably larger absolute number of individuals affected. When compared with Western countries, hypertension among Asian populations has unique features in terms of its onset, clustering of associated cardiovascular risk factors, complications and outcomes. Moreover, only a minority of hypertensive individuals are receiving treatment and achieving control. Projected number of deaths related to hypertension dramatically increased in the last 25 years in some Asian regions with a disproportionately high mortality and morbidity from stroke compared with Western countries. The relation between blood pressure and the risk of stroke is stronger in Asia than in Western regions. Although new Guidelines for hypertension diagnosis and management have been recently released from Europe and North America, the unique features of Asian hypertensive patients raise concerns on the clinical applicability of Western Guidelines to Asian populations. To this purpose, we critically reviewed key elements from the most updated Guidelines. We also discussed their core concepts to verify the impact on hypertension prevention and management in Asian countries.

\section{INTRODUCTION}

Hypertension is a common risk factor for cardiovascular (CV) disease and a serious global health issue, remaining the most powerful predictor of mortality in high-income and low-income countries. ${ }^{1}$ Results from the Global Burden of Disease, Injuries, and Risk Factor study documented that systolic blood pressure (BP) was associated with the highest burden among risk factors, more than either smoking or obesity. ${ }^{1}$ The projected number of individuals with systolic BP of $140 \mathrm{~mm} \mathrm{Hg}$ or higher increased from 442 million $(95 \%$ uncertainty interval (UI) 437 to 447 million) in 1990 to 874 million (95\% UI 864 to 884 million) in 2015, and the associated annual number of projected deaths in 2015 (7.8 million, 95\% UI 7.0 to 8.7 million) or $14.0 \%$ of total deaths $(95 \%$ UI $12.5 \%$ to $15.5 \%)$ and 143 million disability-adjusted life years (95\%
UI 130.2 to 157.0 million) were related to systolic BP of $140 \mathrm{~mm} \mathrm{Hg}$ or higher. ${ }^{1}$

Asia is the world's most populous region and its rapid economic and population growth has brought un-presented burden of chronic disease (including hypertension) and demand for health services to all countries, in particular China and India. ${ }^{2}$

Furthermore, although the average systolic BP is decreasing worldwide since the 1980s at the rate of $1 \mathrm{~mm} \mathrm{Hg}$ systolic BP per decade, it is increasing in low-income and middle-income countries, especially in the South Asian population. ${ }^{23}$ Thus, prevalence of hypertension is increasing in many countries of this region and the much larger base Asian population results in a considerably larger absolute number of individuals affected. ${ }^{4}$

A systematic review of 33 studies from Member Countries of South Asian Association for Regional Cooperation ${ }^{5}$ showed that the overall prevalence of hypertension was around 27\%. Hypertension varied between the studies, ranging between $13.6 \%$ and $47.9 \%$, and was found to be higher in subjects of urban than in rural areas. Prevalence of hypertension from the latest studies ${ }^{5}$ was Bangladesh, 17.9\%; Bhutan, 23.9\%; India, 31.4\%; Maldives, 31.5\%; Mongolia, 47\%; Nepal, 33.8\%; Pakistan, $25 \%$; Republic of Korea, 30\%; and Sri Lanka, $20.9 \% .^{25}$

More recently, data from the Patient-Centred Evaluative Assessment of Cardiac Events (PEACE) Million Persons Project (MPP), ${ }^{6}$ enrolling 1.7 million adults aged $35-75$ years from across China, estimated an age-standardised and sex-standardised prevalence of hypertension (defined as either BP $>140 / 90 \mathrm{~mm} \mathrm{Hg}$ or self-reported use of antihypertensive medication in the previous 2 weeks) equal to $37.2 \%$ (95\% confidence interval [CI] 37.1 to 37.3).

More importantly, only $22.9 \%$ (95\% CI 22.7 to 23.0) of these patients were receiving treatment, and only $5.7 \%$ (95\% CI 5.6 to 5.7 ) were achieving BP control.

In this context, it is worthy to be mentioned that use of medications to prevent and treat $\mathrm{CV}$ disease remains considerably low in $\mathrm{Asia}^{7}$ and most people for whom BP-lowering drugs are clearly indicated receive no treatment whatsoever. ${ }^{28}$

Data from the PEACE MPP study and from a nationwide sample of 3362 primary care facilities ${ }^{9}$ showed that $8.1 \%$ of facilities (95\% CI 7.2 to 9.1 ) did not stock any hypertensive drugs, and only $33.8 \%$ (95\% CI 32.2 to 35.4) stocked all four standard classes of antihypertensive drug classes. Thus, deficiencies in Asian health system make a large contribution to the disease burden. ${ }^{10}$ 
Table 1 Blood pressure categories according to the 2017 American College of Cardiology/American Heart Association (ACC/AHA) and 2018 European Society of Cardiology/European Society of Hypertension (ESC/ESH) Guidelines ${ }^{12} 13$

\begin{tabular}{|c|c|c|c|c|c|c|}
\hline & 2017 ACC/AHA & & & 2018 ESC/ESH & & \\
\hline Category & SBP $(\mathrm{mm} \mathrm{Hg})$ & & $\mathrm{DBP}(\mathrm{mm} \mathrm{Hg})$ & SBP (mm Hg) & & $\begin{array}{l}\mathrm{DBP}(\mathrm{mm} \\
\mathrm{Hg})\end{array}$ \\
\hline Optimal & - & - & & $<120$ & and & $<80$ \\
\hline Normal & $<120$ & and & $<80$ & $120-129$ & and or & $80-84$ \\
\hline High normal & - & - & - & 130-139 & and or & $85-89$ \\
\hline Elevated & $120-129$ & and & $<80$ & - & - & - \\
\hline \multicolumn{7}{|l|}{$\begin{array}{l}\text { Hypertension } \\
\text { stage/grade* }\end{array}$} \\
\hline 1 & 130-139 & or & $80-89$ & 140-159 & and or & $90-99$ \\
\hline 2 & $\geq 140$ & or & $\geq 90$ & $160-179$ & and or & $100-109$ \\
\hline 3 & - & - & - & $\geq 180$ & and or & $\geq 110$ \\
\hline ISH & - & - & - & $\geq 140$ & and & $<90$ \\
\hline
\end{tabular}

* 'Stage' for ACC/AHA; 'grade' for ESC/ESH.

DBP, diastolic blood pressure; ISH, isolated systolic hypertension; SBP, systolic blood pressure.

Finally, the phenotypes of CV disease, stroke and heart failure, which are closely associated with BP, are more common in Asia. ${ }^{11}$ Moreover, the association between hypertension and CV disease is stronger in Asian countries than Western countries ${ }^{11}$ and deaths associated with systolic BP of $140 \mathrm{~mm} \mathrm{Hg}$ or higher significantly increased in the last two decades in Asian regions (East Asia, +151.9\%; Southeast Asia, +101.0\%; Central Asia, $+37.7 \%$; South Asia, $+130.8 \%)^{1}$

\section{Epidemiology of hypertension revised}

New Guidelines for the diagnosis and management of hypertension have been recently released from Europe ${ }^{12}$ and North America. ${ }^{13}$

In the recently released 2018 European Society of Cardiology/ European Society of Hypertension (ESC/ESH) Guidelines, ${ }^{12}$ definition of hypertension remained unchanged (office BP $\geq 140 / 90$ $\mathrm{mm} \mathrm{Hg}$ ) when compared with the previous one (table 1).

Conversely, the 2017 American College of Cardiology/American Heart Association (ACC/AHA) Guidelines for the Prevention, Detection, Evaluation, and Management of High BP in Adults ${ }^{13}$ lowered the systolic and diastolic BP thresholds for diagnosis of hypertension (table 1) to $130 / 80 \mathrm{~mm} \mathrm{Hg}$, a shift that inevitably leads to define more subjects as hypertensive. For example, a cross-sectional analysis from the population-based 2016 Nepal Demographic and Health Survey ${ }^{14}$ documented that when the new definition of hypertension by the ACC/AHA Guidelines $^{13}$ is being adopted, the overall prevalence of hypertension in Nepal would approximately double and the number of individuals requiring pharmacotherapy would increase. Briefly, among 13519 participants recruited, 44.2\% had hypertension according to the 2017 ACC/AHA Guidelines compared with $21.2 \%$ by the Seventh Report of the Joint National Committee on Prevention, Detection, Evaluation, and Treatment of High Blood Pressure Guidelines. ${ }^{14}$ When estimating the proportion of hypertension by background characteristics, the new 2017 ACC/ AHA Guidelines definition increased the prevalence to $50 \%$ or greater for some categories, with the highest prevalence among those with a body mass index greater than or equal to 30 and between 25 and $29.9 \mathrm{~kg} / \mathrm{m}^{2}$.

Similarly, Khera and coworkers estimated the relative increase of prevalence among adults aged 45 to 75 years in China and found an overall relative increase of $26.8 \%$ in this country ${ }^{15}$ (figure 1).

It has been also reported that CV events were often diagnosed at lower BP levels than the $140 / 90 \mathrm{~mm} \mathrm{Hg}$ cut-off points in some
Asian regions, questioning whether appropriate (and lower) limit values of BP to define hypertension should be used, especially for developing country populations. ${ }^{16}$

Of note, epidemiological studies on hypertension in western Indian urban and rural cohorts ${ }^{17}$ showed that distributions of BP are different in various age groups; for example, in men aged 20-29 years, a systolic BP of $140 \mathrm{~mm} \mathrm{Hg}$ is not reached until the 95th percentile while at 60-69 years it is achieved at the 70th percentile. ${ }^{17}$ Similar variations are seen in diastolic BP. ${ }^{17}$ In other words, either percentile values for diagnosis of hypertension should be different at various age groups or the systolic/ diastolic BP for the diagnosis of hypertension should be lower in younger subjects.

\section{Impact of 'SPRINT'}

The 2017 ACC/AHA Guidelines for the Prevention, Detection, Evaluation, and Management of High BP in Adults ${ }^{13}$ recommend a systolic BP target $<130 \mathrm{~mm} \mathrm{Hg}$ in almost all hypertensive patients. They fully endorse the evidence of benefit for a lower systolic BP target than that currently recommended, as documented in the Systolic Blood Pressure Intervention Trial (SPRINT). ${ }^{18}$

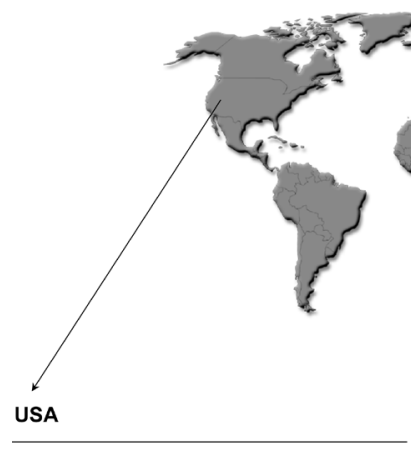

Prevalence of hypertension:

70.1 million people (increase $26.8 \%$ )

Candidates for intensification of treatment: increase by 13.9 million

(from $24.0 \%$ to $54.4 \%$ of treated patients)

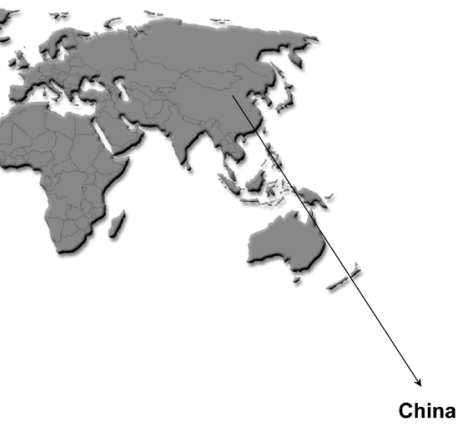

Prevalence of hypertension: 266.9 million people (increase $45.1 \%$ )

Candidates for intensification of treatment: increase by 30.0 million (from $41.4 \%$ to $76.2 \%$ of treated patients)
Figure 1 Effects of the 2017 American College of Cardiology/ American Heart Association hypertension guidelines on the prevalence of hypertension and eligibility for initiation and intensification of treatment in nationally representative populations from the USA and China. Data from Khera et al. ${ }^{15}$ 
Briefly, the SPRINT trial included 9361 participants with a systolic BP of $130 \mathrm{~mm} \mathrm{Hg}$ or higher and an increased CV risk randomly assigned to a systolic BP target of $<120 \mathrm{~mm} \mathrm{Hg}$ (intensive treatment) or a target of $<140 \mathrm{~mm} \mathrm{Hg}$ (standard treatment).

Findings indicated that patients in the intensive treatment group had a $25 \%$ risk reduction in the primary composite end point of major CV events (eg, myocardial infarction, nonmyocardial infarction acute coronary syndrome, stroke, acute decompensated heart failure and CV disease death) and a 27\% risk reduction in mortality compared with those who received standard treatment. ${ }^{18}$

Of note, the SPRINT trial ${ }^{18}$ used programmable automated oscillometric devices to measure BP. Nonetheless, there is confusion in recent publications regarding whether the BP measurements at SPRINT clinics were unattended. ${ }^{19}$ Concern has also been expressed that the BP readings obtained in SPRINT were not comparable with $\mathrm{BP}$ readings in other trials where the measurement was attended and that the intensive treatment goal of $<120 \mathrm{~mm} \mathrm{Hg}$ in SPRINT would actually correspond to higher systolic BP values in other trials. ${ }^{19} 20$

As pointed out by a recent report by Johnson and coworkers, ${ }^{20}$ there were 4082 participants at 38 sites that measured BP after leaving the participant alone the entire time (always alone), 2247 at 25 sites that had personnel in the room the entire time (never alone), 1746 at 19 sites that left the participant alone only during the rest period (alone for rest) and 570 at 6 sites that left the participant alone only during the BP readings (alone for BP measurement). Importantly, similar BP levels and CV disease risk reduction were observed in the intensive group in SPRINT participants whether the measurement technique used was primarily attended or unattended.

Although the available evidence clearly supports the notion that even a small decrease in $\mathrm{BP}$ reduces risks of $\mathrm{CV}$ morbidity and mortality ${ }^{21}$ (figure 2) and that an intensive BP-lowering strategy significantly reduces the risk of $\mathrm{CV}$ mortality without evidence of harm (figure 3), ${ }^{22}$ the ESC/ESH Guidelines stated that BP should be lowered to levels $<140 / 90 \mathrm{~mm} \mathrm{Hg}$ in all patients (I A recommendation) and to $\leq 130 / 80 \mathrm{~mm} \mathrm{Hg}$ 'in
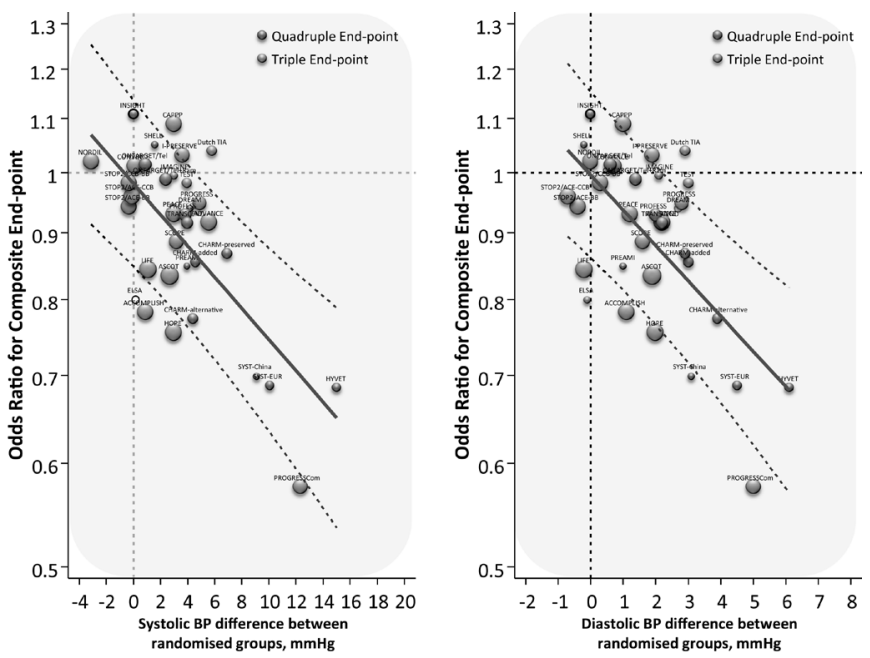

Figure 2 Relationship between the reduction in blood pressure (BP) and the risk of composite cardiovascular endpoints (triple: myocardial infarction, stroke and cardiovascular death; quadruple: myocardial infarction, stroke, cardiovascular death and congestive heart failure). Thirty trials are included for a total of 221024 patients. For each trial, the difference in BP between randomised groups is depicted. Data from Verdecchia et $\mathrm{al}^{21}$ (with permission). BP, blood pressure.

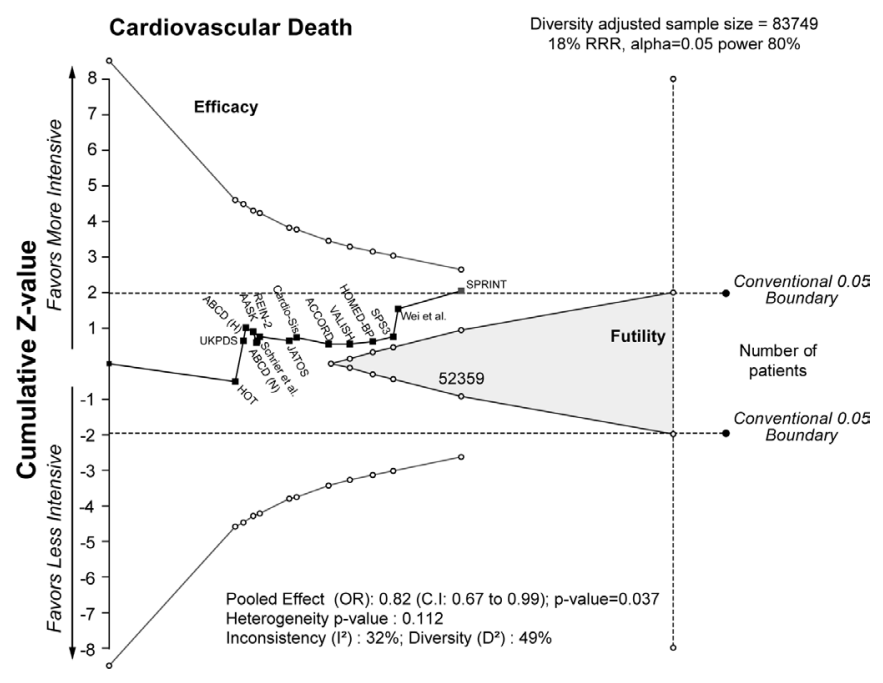

Figure 3 Trial sequential analysis (TSA) of the effect of more intensive vs less intensive blood pressure reduction on cardiovascular mortality. See Verdecchia et $a^{22}$ for guidance on TSA and plot interpretation (with permission). $\mathrm{RRR}$, relative risk reduction; $\mathrm{OR}$, odds ratio; $\mathrm{Cl}$, confidence interval.

most patients' provided that the treatment is 'well tolerated' (I A recommendation). ${ }^{12}$ Nevertheless, the European Guidelines ${ }^{12}$ recommend that systolic BP should be lowered (1) to $<140$ $\mathrm{mm} \mathrm{Hg}$, but not to $<130 \mathrm{~mm} \mathrm{Hg}$, in patients aged $\geq 65$ years and $(2)<130 \mathrm{~mm} \mathrm{Hg}$, but not below $120 \mathrm{~mm} \mathrm{Hg}$ in younger patients (<65 years; I A recommendation). ${ }^{12}$ Similarly, 2018 ESC/ESH European Guidelines ${ }^{12}$ recommend to lower systolic $\mathrm{BP}$ to a range 130 to $<140 \mathrm{~mm} \mathrm{Hg}$ in patients with diabetic or non-diabetic chronic kidney disease. ${ }^{23}$

In other words, the European Guidelines ${ }^{12}$ support the clinical use of 'safety boundaries', not to be crossed towards lower BP levels even in patients with a perfect tolerability of treatment.

\section{Safety boundaries in the Asian context}

Lowering the threshold for diagnosis of hypertension, as recommended by the ACC/AHA Guidelines, may be considered a valuable achievement in the light of evidence that a higher BP is associated with adverse CV outcomes even at levels below 140/90 $\mathrm{mm} \mathrm{Hg}{ }^{13}$.

An analysis of an ongoing cluster-randomised community-based screening trial from India demonstrated that BP was associated with CV mortality at thresholds lower than the ESC/ ESH hypertension definition. ${ }^{16}$ Specifically, 167331 adults aged 35-90 years were included in the analysis. Death rates were calculated according to systolic and diastolic BP, and their associations were examined in multivariable analyses. The nadir of $\mathrm{CV}$ death rates was observed at $110 \mathrm{~mm} \mathrm{Hg}$ for casual systolic $\mathrm{BP}$ and $75-80 \mathrm{~mm} \mathrm{Hg}$ for casual diastolic BP. In the multivariable analyses, systolic BP from $120 \mathrm{~mm} \mathrm{Hg}$ and diastolic BP from $90 \mathrm{~mm} \mathrm{Hg}$ were significantly associated with risks of ischaemic heart disease and stroke.

Furthermore, in the Mumbai Cohort Study, pre-stage hypertension (systolic BP 130-139 and/or diastolic BP 85-89 mm Hg) was associated to an increased risk of cerebrovascular death in men (hazard ratio [HR] 1.73 , 95\% CI 1.15 to 2.61 ) when compared with normal BP (systolic BP $<120$ and diastolic BP $<80 \mathrm{~mm} \mathrm{Hg})^{24}$

More recently, a prospective study of 5752 subjects free of CV disease (Chinese Multi-provincial Cohort Study) ${ }^{25}$ evaluated to what extent the new BP stratum of stage 1 hypertension 
(systolic/diastolic BP of 130 to $139 / 80$ to $89 \mathrm{~mm} \mathrm{Hg}$ ) affects the $\mathrm{CV}$ risk. The participants with baseline systolic/diastolic BP $<130 / 80 \mathrm{~mm} \mathrm{Hg}, 130-139 / 80-89 \mathrm{~mm} \mathrm{Hg}$ and $\geq 140 / 90 \mathrm{~mm} \mathrm{Hg}$ were 2892 (50.3\%), 1328 (23.1\%) and 1532 (26.6\%), respectively. After adjustment for several confounders (including age, sex, body mass index, smoking status, diabetes, total cholesterol and high-density lipoprotein cholesterol at baseline), participants who maintained a systolic/diastolic BP at 130-139/80-89 $\mathrm{mm} \mathrm{Hg}$ during follow-up had a higher risk of developing acute $\mathrm{CV}$ events, coronary heart disease and stroke with the HRs of 2.04 (95\% CI 1.16 to 3.57$), 3.29$ (95\% CI 1.30 to 8.35 ) and 1.63 (95\% CI 0.80 to 3.33 ), compared with those who maintained their systolic BP $<130 \mathrm{~mm} \mathrm{Hg}$ and diastolic BP $<80$ $\mathrm{mm} \mathrm{Hg}$. Participants whose BP increased from 130-139/80-89 $\mathrm{mm} \mathrm{Hg}$ to hypertension over the follow-up period had 2.81-fold (95\% CI 1.84 to 4.29 ), 3.17-fold (95\% CI 1.43 to 7.03 ) and 2.71-fold (95\% CI 1.65 to 4.44 ) higher risk for the incidence of acute CV events, coronary heart disease and stroke, respectively, compared with participants who maintained their systolic BP $<130 \mathrm{~mm} \mathrm{Hg}$ and diastolic BP $<80 \mathrm{~mm} \mathrm{Hg}$.

Overall, the BP targets recommended by the ACC/AHA Guidelines ${ }^{13}$ considerably differ from the indications released by the ESC/ESH Guidelines. ${ }^{12}$

Furthermore, the use of 'safety boundaries', as suggested by the European Guidelines, ${ }^{12}$ raises important concerns in the treatment of hypertension in Asian populations.

A first consideration regards the management of elderly subjects. With a rapidly ageing population, the prevalence of hypertension in Asia continues to rise, and older adults account for the bulk of hypertension-related morbidity and mortality. ${ }^{24}$ In many Asian countries, hypertension is very common in the elderly. For example, the prevalence of hypertension in China is $39 \%$ overall, $59.4 \%$ in patients aged $\geq 60$ years and $72.8 \%$ in those aged $\geq 75$ years. $^{26}$ Although none of the available Asian intervention trials provided strong support for more ambitious BP targets in elderly patients with hypertension, ${ }^{2}$ a recent meta-analysis by Yano and coworkers ${ }^{27}$ showed a benefit in terms of CV and stroke prevention when a BP target of less than $140 / 80 \mathrm{~mm} \mathrm{Hg}$ is achieved in Asian hypertensive patients. More specifically, in the subgroup analysis of elderly patients, stroke risk was decreased by intervention in trials comparing an active treatment with a placebo, or a more intensive with less intensive $\mathrm{BP}$ control (odds ratio [OR] 0.74, 95\% CI 0.58 to 0.93 ). ${ }^{27}$

In this context, Asian physicians need to consider specific features of their hypertensive patients ${ }^{4}$ regarding the association between elevated BP and the risk of CV disease. ${ }^{28}$ The Asia Pacific Cohort Studies Collaboration, which involved $>500000$ individuals followed for several years, has demonstrated ${ }^{28}$ that the association of BP with the risk of stroke was about twice as steep as that with coronary heart disease risk. While the association with coronary risk was similar in the Asian and Caucasian populations, the association with stroke risk was steeper among Asians. $^{28}{ }^{29}$ Moreover, in the aforementioned meta-analysis by Yano and coworkers, ${ }^{27}$ a $10 \mathrm{~mm} \mathrm{Hg}$ reduction in systolic BP was associated with a $30 \%$ reduced risk for stroke. ${ }^{27}$

Taken together, these findings suggest that the clinical use of 'safety boundaries' (ie, treated systolic BP not below $130 \mathrm{~mm} \mathrm{Hg}$ in subjects aged 65 years or more, and not below $120 \mathrm{~mm} \mathrm{Hg}$ in subjects aged less than 65 years), as recommended by the European Guidelines, ${ }^{12}$ might be counterproductive in Asia. Implementation of 'safety boundaries' could induce doctors to refrain from achieving lower BP targets that, if well tolerated, would reasonably result in a lesser risk of stroke, so frequent in Asia, and other major complications of hypertension.

\section{CONCLUSIONS}

The high prevalence of hypertension in Asia notably contributes to the growing worldwide burden of CV disease and death. ${ }^{2}$ The Asia Pacific Cohort Studies Collaboration clearly showed a progressive reduction in the risk of stroke, ischaemic heart disease and CV death up to BP values of about 115/75 $\mathrm{mm} \mathrm{Hg} .{ }^{28}$ Notably, such relation held in both genders, and a 10 $\mathrm{mm} \mathrm{Hg}$ lower systolic BP was associated with a 41\% lower risk of stroke. ${ }^{28}$ Similar results were also obtained from the aforementioned Trivandrum community-based study ${ }^{16}$ and Mumbai Cohort Study. ${ }^{24}$

Being stroke a dominant complication of hypertension in Asia, the substantial benefit of an intensive BP reduction on the risk of stroke should be considered in future Guidelines on the management of hypertension in Asian populations.

In general, adaptation of western Hypertension Guidelines to the Asian context is not automatic. The use of relatively low diagnostic thresholds and therapeutic targets (130/80 $\mathrm{mm} \mathrm{Hg}$ ), as suggested by the ACC/AHA Guidelines, ${ }^{13}$ appears acceptable in an Asian context. Conversely, the message of European Guidelines ${ }^{12}$ should be probably applied with more caution. The European recommendation ${ }^{12}$ that systolic BP should not be lowered $<130 \mathrm{~mm} \mathrm{Hg}$ in patients aged $\geq 65$ years and $<120 \mathrm{~mm} \mathrm{Hg}$ in patients aged $<65$ years is not supported by at least three well-conducted systematic reviews and meta-analyses. In a meta-regression analysis by Ettehad and coworkers, a greater reduction in systolic BP was associated with a greater risk reduction without any evidence of a J-curve effect. ${ }^{30}$ In a network meta-analysis by Bundy and coworkers, a mean achieved systolic BP 120-124 mm Hg was associated with a significant reduction in the risk of cardiovascular disease and all-cause mortality even in the comparison with achieved systolic BP levels $125-129 \mathrm{~mm} \mathrm{Hg} .{ }^{31}$ Bangalore and coworkers published a network meta-analysis of trials designed to compare different BP targets and concluded that systolic BP targets $<120 \mathrm{~mm} \mathrm{Hg}$ and $<130 \mathrm{~mm} \mathrm{Hg}$ ranked number 1 and number 2, respectively, as the most efficacious for preventing stroke and myocardial infarction. ${ }^{32}$ Conversely, systolic BP targets $<140 \mathrm{~mm} \mathrm{Hg}$ and $<150 \mathrm{~mm} \mathrm{Hg}$ ranked as number 1 and number 2, respectively, for safety. Overall, a systolic BP target $<130 \mathrm{~mm} \mathrm{Hg}$ achieved the best balance between efficacy and safety. ${ }^{32}$

In summary, the use of $130 / 80 \mathrm{~mm} \mathrm{Hg}$ as a diagnostic threshold and management goal of BP may contribute to more sustained target organ protection and CV disease prevention. Instead of fixing rigid BP targets or safety boundaries, what we should pursue in the daily practice is an optimal balance between the magnitude of achieved BP reduction and the tolerability of treatment in the single patient. ${ }^{33}$ An aphorism might be: 'The lowest well-tolerated blood pressure, the better'. This conclusion seems to particularly applicable to Asia, where the achievement of normal or optimal BP levels for prevention of stroke remains a priority in the treatment of hypertension.

Acknowledgements Supported in part by the non-profit foundation 'Fondazione Umbra Cuore e Ipertensione-ONLUS', Perugia, Italy.

Contributors All authors have read and approved the manuscript.

Funding The authors have not declared a specific grant for this research from any funding agency in the public, commercial or not-for-profit sectors.

Competing interests None declared.

Patient consent for publication Not required.

Provenance and peer review Not commissioned; externally peer reviewed. 


\section{REFERENCES}

1. Forouzanfar $M H$, Liu $P$, Roth GA, et al. Global burden of hypertension and systolic blood pressure of at least 110 to $115 \mathrm{~mm} \mathrm{Hg}$, 1990-2015. JAMA 2017;317:165-82

2. Angeli F, Reboldi G, Verdecchia P. The 2014 hypertension guidelines: implications for patients and practitioners in Asia. Heart Asia 2015;7:21-5.

3. Danaei G, Finucane MM, Lin JK, et al. National, regional, and global trends in systolic blood pressure since 1980: systematic analysis of health examination surveys and epidemiological studies with 786 country-years and 5.4 million participants. Lancet 2011;377:568-77.

4. Chung N, Baek S, Chen M-F, et al. Expert recommendations on the challenges of hypertension in Asia. Int J Clin Pract 2008;62:1306-12.

5. Neupane D, McLachlan CS, Sharma R, et al. Prevalence of hypertension in member countries of South Asian association for regional cooperation (SAARC): systematic review and meta-analysis. Medicine 2014;93:e74.

6. Lu J, Lu Y, Wang X, et al. Prevalence, awareness, treatment, and control of hypertension in China: data from 1.7 million adults in a population-based screening study (China peace million persons project). Lancet 2017;390:2549-58.

7. Chow CK, Teo KK, Rangarajan S, et al. Prevalence, awareness, treatment, and control of hypertension in rural and urban communities in high-, middle-, and low-income countries. JAMA 2013;310:959-68.

8. Perkovic V, Huxley R, Wu Y, et al. The burden of blood pressure-related disease: a neglected priority for global health. Hypertension 2007;50:991-7.

9. Su M, Zhang Q, Bai X, et al. Availability, cost, and prescription patterns of antihypertensive medications in primary health care in China: a nationwide crosssectional survey. Lancet 2017;390:2559-68.

10. Hesketh T, Zhou X. Hypertension in China: the gap between policy and practice. Lancet 2017;390:2529-30.

11. Kario K, Chen C-H, Park S, et al. Consensus document on improving hypertension management in Asian patients, taking into account Asian characteristics. Hypertension 2018;71:375-82.

12. Williams B, Mancia G, Spiering W, et al. 2018 practice guidelines for the management of arterial hypertension of the European Society of Hypertension and the European Society of Cardiology: ESH/ESC Task Force for the management of arterial hypertension. J Hypertens 2018;36:2284-309.

13. Whelton PK, Carey RM, Aronow WS, et al. 2017 ACC/AHA/AAPA/ABC/ACPM/AGS/ APhA/ASH/ASPC/NMA/PCNA guideline for the prevention, detection, evaluation, and management of high blood pressure in adults: a report of the American College of Cardiology/American Heart Association Task Force on Clinical Practice Guidelines. $J$ Am Coll Cardiol 2018;71:e127-248.

14. Kibria GMA, Swasey K, Kc A, et al. Estimated change in prevalence of hypertension in Nepal following application of The 2017 ACC/AHA guideline. JAMA Netw Open 2018;1:e180606.

15. Khera R, Lu Y, Lu J, et al. Impact of 2017 ACC/AHA guidelines on prevalence of hypertension and eligibility for antihypertensive treatment in United States and China: nationally representative cross sectional study. BMJ 2018;362.

16. Sauvaget C, Ramadas K, Thomas G, et al. Prognosis criteria of casual systolic and diastolic blood pressure values in a prospective study in India. J Epidemiol Community Health 2010;64:366-72.
17. Gupta R. Defining hypertension in the Indian population. Nat/ Med J India 1997;10:139-43.

18. Wright JT, Williamson JD, Whelton PK, et al. A randomized trial of intensive versus standard blood-pressure control. N Engl J Med 2015;373:2103-16.

19. Kjeldsen SE, Lund-Johansen P, Nilsson PM, et al. Unattended blood pressure measurements in the systolic blood pressure intervention trial: implications for entry and achieved blood pressure values compared with other trials. Hypertension 2016;67:808-12.

20. Johnson KC, Whelton PK, Cushman WC, et al. Blood pressure measurement in sprint (systolic blood pressure intervention trial). Hypertension 2018;71:848-57.

21. Verdecchia P, Gentile G, Angeli F, et al. Influence of blood pressure reduction on composite cardiovascular endpoints in clinical trials. J Hypertens 2010;28:1356-65.

22. Verdecchia P, Angeli F, Gentile G, et al. More versus less intensive blood pressurelowering strategy: cumulative evidence and trial sequential analysis. Hypertension 2016;68:642-53.

23. Reboldi G, Gentile G, Angeli F, et al. The 2018 ESC/ESH hypertension guidelines: should nephrologists always stop at the lower boundary? J Nephrol 2018;31:621-6.

24. Pednekar MS, Gupta R, Gupta PC. Association of blood pressure and cardiovascular mortality in India: Mumbai cohort study. Am J Hypertens 2009;22:1076-84.

25. Han XY, Qi Y, Zhao D, et al. [Association between long-term blood pressure change and the incidence of cardiovascular diseases: a population-based cohort study]. Zhonghua Xin Xue Guan Bing Za Zhi 2018;46:695-700.

26. Sheng C-S, Liu M, Kang Y-Y, et al. Prevalence, awareness, treatment and control of hypertension in elderly Chinese. Hypertens Res 2013;36:824-8.

27. Yano Y, Briasoulis A, Bakris GL, et al. Effects of antihypertensive treatment in Asian populations: a meta-analysis of prospective randomized controlled studies (CARdiovascular protectioN group in Asia: (ARNA). J Am Soc Hypertens 2014;8:103-16.

28. Lawes CMM, Rodgers $A$, Bennett DA, et al. Blood pressure and cardiovascular disease in the Asia Pacific region. J Hypertens 2003;21:707-16.

29. Ishikawa Y, Ishikawa J, Ishikawa S, et al. Prehypertension and the risk for cardiovascular disease in the Japanese general population: the Jichi Medical School Cohort Study. J Hypertens 2010;28:1630-7.

30. Ettehad D, Emdin CA, Kiran A, et al. Blood pressure lowering for prevention of cardiovascular disease and death: a systematic review and meta-analysis. Lancet 2016;387:957-67.

31. Bundy JD, Li C, Stuchlik P, et al. Systolic blood pressure reduction and risk of cardiovascular disease and mortality: a systematic review and network meta-analysis. JAMA Cardiol 2017;2:775-81.

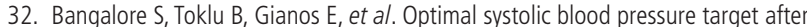
sprint: insights from a network meta-analysis of randomized trials. Am J Med 2017:130:707-19.

33. Verdecchia P, Angeli F, Cavallini C, et al. Keep blood pressure low, but not too much... does evidence support the recommendation of rigid "safety boundaries" ? Circulation Research 2018;123:1205-7.

34. Fuster V. No such thing as ideal blood pressure: a case for personalized medicine. $J$ Am Coll Cardiol 2016;67:3014-5. 